\title{
Gesù Nazareno. Un colloquio con Mauro Pesce. A cura di Cristiana Facchini
}

\section{Mauro Pesce}

Storicamente, 2 (2006).

ISSN: 1825-411X. Art. no. 61. DOI: 10.12977/stor543

II mio libro Le parole dimenticate di Gesù (Mondadori-Lorenzo Valla) del settembre 2004 aveva avuto un successo inaspettato nonostante si trattasse di una voluminosa antologia in greco e in latino con un commento molto tecnico. Ho intuito perciò che un vasto pubblico aveva un interesse a conoscere i risultati scientifici delle indagini storiche su Gesù. Quando Augias mi propose un'intervista su Gesù ero quindi in qualche modo già pronto ad accettare.

Chiedendomi quale sia il rapporto tra domande e risposte tu metti il dito sulla questione centrale del libro. Augias non ha fatto domande sue, ma, da grande professionista quale è, si è fatto portavoce delle domande che - a suo giudizio - erano le più diffuse e sentite in un vasto pubblico. In un processo di divulgazione è fondamentale innestare i risultati della ricerca scientifica sul bisogno reale della gente. Non basta esprimersi in modo chiaro e semplice. È necessaria una accurata conoscenza della situazione culturale in cui la maggioranza della popolazione vive. C'è poi bisogno di un meccanismo di promozione che segnali il prodotto-libro a vasti strati della popolazione, in modo da suscitare interesse verso la lettura.

Devo però dire che il successo del libro ha per me anche un aspetto fastidioso, perché le cose che dico nelle mie risposte ad Augias erano già state da me esposte varie volte in una quantità di articoli e libri scientifici che non hanno riscosso un così vasto interesse. Questo fatto esige risposte 
pratiche sui modi di trasmissione del sapere nella società di oggi.

Le polemiche scaturite dalla pubblicazione di questo libro sono sconcertanti, lasciano intravvedere una cultura italiana arretrata, una Chiesa cattolica arroccata su posizioni difensive d'altri tempi. Molti di noi hanno pensato alla figura di Ernest Renan, allo scandalo suscitato dalla pubblicazione di quel libretto La vie de Jesus che fu un bestseller ottocentesco. Ma questo accadeva quasi un secolo e mezzo fa. Perché secondo te questo libro su Gesù in un'Italia tutto sommato moderna, ha suscitato un dibattito così acceso e molto spesso non privo di mistificazioni gratuite?

Il fatto che «Avvenire», il giornale che esprime il parere della Conferenza Episcopale Italiana, sia intervenuto per ben tre volte sul libro e che addirittura "Civiltà cattolica» abbia pubblicato un articolo di condanna dogmatica è sintomo del fatto che una certa parte del cattolicesimo italiano si è spaventato. «Avvenire» ha mobilitato il padre Cantalamessa, molto noto per la sua costante presenza televisiva, affinché un vasto pubblico cattolico avesse una riposta rassicurante contro gli eventuali pericoli per la fede che il libro a suo parere poteva costituire. L'articolo del padre De Rosa su «Civiltà cattolica» ha invece la funzione - credo - di avvisare in modo indiretto, ma molto chiaro, gli esegeti cattolici, soprattutto sacerdoti, su cosa potrebbe succedere loro se seguissero il mio esempio. Sarebbero condannati, esclusi dall'insegnamento e le case editrici cattoliche dirette da sacerdoti e ordini religiosi sarebbero ugualmente sottoposte a censura. 
Quello che colpisce negli articoli di Cantalamessa e De Rosa è il fatto che non presentano correttamente il mio pensiero e anche che cerchino di denigrarmi sul piano professionale. Per questo motivo chiedo a «Storicamente» di inserire come appendice a questa intervista la mia risposta dettagliata a cantalamessa e a De Rosa. Si tratta di risposte lunghe e tecniche, che sono però necessarie per comprendere la natura di questi due attacchi ecclesiastici.

Bisogna dire tuttavia che questi due attacchi non rappresentano affatto tutta la Chiesa cattolica, ma solo due orientamenti romani. Le Edizioni Paoline hanno assunto un ben altro atteggiamento su «Letture», «Vita pastortale» e su «Jesus». In nessuno di questi giornali si troverà una condanna. Devo ringraziare le redazioni di «Letture» e de «ll regno» (quest'ultimo delle Edizioni Dehoniane di Bologna) per avere trascorso con me alcune ore rispettivamente a Milano e Bologna discutendo e presentandomi obiezioni anche serie, ma sempre rispettose. Molti sacerdoti e parroci hanno avuto reazioni favorevoli. Cantalamessa - che è persona certo molto intelligente e preparata - mi sembra invece farsi carico della posizione dei movimenti ecclesiali, i quali, mettono, sì, al centro della loro vita religiosa, la Bibbia e quindi il Nuovo Testamento, ma senza un'adeguata preparazione esegetica (da parte dei membri dei movimenti) e corrono perciò il pericolo di cadere a volte in tendenze fondamentaliste. II Padre De Rosa mi sembra al contrario più vicino alle preoccupazioni teologiche del cardinal Ruini e di ambienti teologici romani conservatori o neoconservatori che tendono a coprirsi con le idee teologiche dell'attuale Papa.

La ricerca sulla vita di Gesù - ma per esteso sulla storia del cristianesimo e sulle religioni - rimane in questo paese ancora un affare di pochi. Quando questi temi escono dall'accademia, dagli ambiti ristretti del dibattito scientifico internazionale, allora emergono due posizioni nette: da un lato il plauso acritico dei "laici", accompagnato talvolta da un assoluto disinteresse, 0 le accuse animose di esponenti ecclesiastici, coadiuvato dagli intellettuali cattolici organici, quei laici cioè che si adoperano in modi differenti per diffondere capillarmente le posizioni ufficiali della Chiesa cattolica. 


\section{Sembra che in questo paese la riflessione autonoma, indipendente su} tematiche di carattere religioso sia prerogativa di pochi. Perché la Chiesa cattolica teme la libertà di pensiero su questioni religiose?

Perché la Chiesa cattolica tema la libertà di pensiero su questioni religiose è una domanda a cui temo di non avere una risposta adeguata. Anzitutto, come ho fatto con la domanda precedente, non parlerei di Chiesa cattolica in generale. Si tratta soltanto di alcuni ambienti ecclesiastici romani. La Chiesa cattolica rappresenta nel mondo di oggi una realtà estremamente variegata, ricca di esperienze straordinarie in ogni parte del pianeta, inclusa I'Italia, straordinarie per il loro valore etico, umano e religioso. Anche in Italia, negli ordini religiosi, nelle parroccchie, tra la gente di fede cattolica esiste una ricchissima varietà di posizioni che sono lungi dall'identificarsi con alcune tendenze teologiche e politiche neoconservatrici romane.

Detto questo, è però vero che una certa tendenza tipicamente cattolica a delegare ai soli sacerdoti il sapere e ai laici una posizione intellettualmente subordinata non è stata realmente modificata dal Concilio Vaticano II nonostante la sua insistenza sul "sacerdozio universale dei fedeli". Ciò significa che i sacerdoti ricevono una formazione esegetica spesso approfondita, ma non i laici i quali perciò non hanno strumenti adeguati per comprendere l'analisi storica dei testi biblici e della figura storica di Gesù. Da qui la preoccupazione della gerarchia di impedire il più possibile una divulgazione pubblica dei dibattiti esegetici che obbligherebbe ad un divcrso rapporto clero-laicato.

Alcuni hanno forse pensato che per porre rimedio al fatto che qualche centinaia di migliaia di persone aveva letto il libro Inchiesta su Gesù, era opportuno denigrare l'esegeta Mauro Pesce dal punto di vista scientifico e dottrinale in modo da togliergli autorità rispetto al pubblico cattolico. Ma il punto è che le tesi storiche che io espongo sono largamente diffuse nell'esegesi internazionale. Il passaggio dalla ricerca storica alla visione di fede richiede numerosi passaggi intermedi. L'esegesi studia $i$ testi dei 
vangeli uno per uno, non come parte di una collezione canonica ispirata da Dio. Per questo motivo, nelle Facoltà teologiche, dopo l'esegesi si accede ad un diverso insegnamento che è quello della teologia biblica che parte dall'insieme del Nuovo Testamento. I dati della teologia biblica vengono poi assunti ad un terzo livello ulteriore, quello della teologia dogmatica, che a sua volta diviene la base per la teologia pastorale e per la teologia morale, che più direttamente investono la vita concreta dei fedeli. Alcune autorità ecclesiastiche temono che un contatto diretto della gente con l'esegesi storica metta in crisi quella serie di passaggi ermeneutici e teologici che permette alla Chiesa di fondare il proprio attuale assetto. Ma per ovviare a questo pericolo basterebbe una maggiore informazione.

Quando si discute di Gesù si capisce con facilità che molte delle direttive del Vaticano II sono rimaste lettera morta. È chiaro che quella stagione storica è finita, e forse non ha neanche raggiunto gli obiettivi che si erano posti i padri conciliari. II problema del Gesù storico apre una difficile questione di rapporti con l'ebraismo. Perché ancora suscita scandalo il Gesù ebreo?

Permettimi di rispondere in due tempi. II Cardinal Ruini, nella sua posizione di grande rilievo ecclesiastico, ha cercato di condannare la lettura storica che del Concilio Vaticano II ha dato la Storia del Concilio diretta da Giuseppe Alberigo e ha proposto una sua lettura di questo evento che tende a negarne sostanzialmente l'innovazione. Dal punto di vista degli studi biblici, il fatto certamente positivo, di avere messo dopo tanti secoli, la Bibbia al centro della vita della Chiesa ha avuto anche effetti negativi. L'effetto negativo principale sta nel fatto che nel momento in cui la Chiesa ha messo finalmente la Bibbia a contatto diretto con il popolo fedele, ha però cercato di marginalizzare l'esegesi storico-scientifica perché troppo difficile e pericolosa per i fedeli. Accusata di aridità e di non fornire sufficiente nutrimento religioso, l'esegesi storica è stata sostituita spesso da un'esegesi "spirituale", a volte ispirata all'interpretazione allegorica degli antichi Padri della Chiesa, a volte semplicemente moraleggiante ed intimistica. II pericolo 
per la teologia biblica postconciliare è di separarsi troppo dallo stato attuale della ricerca scientifica. Credo che oggi lo scollamento di molti ambienti ecclesiastici dalla ricerca scientifica, non solo esgetica e storica, sia considerevole. E ciò crea un pericolo di una certa involuzione della Chiesa cattolica.

Quanto alla questione ebraica, è certo vero che la chiesa cattolica dal 1965 ad oggi ha prodotto un numero strordinario di documenti in cui vengono radicalmente criticati i presupposti dell'anti-ebraismo e dell'antisemitismo cristiano dei secoli passati. È però anche vero che questi documenti hanno avuto scarso impatto sulla popolazione dei fedeli e tra gli stessi teologi. In Inchiesta su Gesù, una delle mie affermazioni più contestate da Cantalamessa e De Rosa è proprio quella che Gesù è un ebreo e non un cristiano. Ciò è ovvio nella letteratura esegetica, ma non per loro. In realtà le affermazioni contenute ad esempio nei Sussidi per una corretta presentazione degli Ebrei e dell'Ebraismo nella catechesi e nella predicazione della chiesa cattolica del 1985 o anche molti paragrafi del Catechismo della Chiesa cattolica sono rimasti per lo più lettera morta e i fedeli non li conoscono. Quello che io dico nel libro sull'ebraicità di Gesù è già sostanzialmente in questi documenti cattolici ufficiali.

Sei stato accusato di avere privilegiato, nella tua ricerca su Gesù, i testi apocrifi del Nuovo Testamento contro quelli canonici. Ma dal libro questo non emerge per nulla. Anzi oserei dire che molta attenzione è dedicata al Gesù del Vangelo di Giovanni, a cui tu a hai dedicato molti studi. Mi ha molto colpito quel Gesù così intensamente mistico. Vorresti spiegare come sei giunto a decodificare quelle che tu chiami «le esperienze religiose» dell'uomo Gesù? Come è possibile, e non solo per uno storico dell'antichità, ricostruire le forme dell'esperienza individuale di una personalità religiosa del calibro di Gesù? Vorrei che tu spiegassi le procedure metodologiche che utilizzi per analizzare un testo religioso. 
È vero che il Gesù religioso e mistico è un aspetto che ho voluto sottolineare in modo particolare e a cui tengo molto. Come posso essere certo di potere ricostruire storicamente in modo attendibile le esperienze religiose di Gesù? Quali sono le mie procedure metodologiche? Rispondo sommariamente. Più che i diversi criteri di storicità elaborati da tanti esegeti, per me è fondamentale distinguere gli elementi chiaramente attribuibili alla redazione di un evangelista rispetto alla tradizione che egli elabora: si tratta di un criterio negativo. Ciò che è tipico di un evangelista è sua creazione o creazione del suo ambiente (anche se resta il problema di chiarire come questa innovazione si radichi sulla tradizione precedente). Questa distinzione permette di fare passi indietro verso tradizioni più antiche e più originali. È a questo punto che la convergenza tra tradizioni antiche adddita strati più vicini a Gesù stesso. Qui diventa estremamente importante chiarire i modi di trasmissione di questi materiali antichi, la molteplice attestazione di fonti indipendenti, le forme culturali. Alla base della mia ricerca sta non tanto la cosiddetta terza ricerca su Gesù, quanto invece uno studio socioantropologico delle fonti che rintraccia anzitutto i presupposti culturali degli strati profondi dei testi, poi gli ambienti dei discepoli di Gesù da cui il testo proviene (secondo livello del testo) senza concentrarsi soltanto sul terzo livello esplicito del testo. Sono queste forme culturali — che ho studiato ad esempio nell'ultimo libro Forme culturali del cristianesimo nascente (Brescia, Morcelliana 2006) scritto a quattro mani con Adriana Destro - che permettono di confrontare le esperienze religiose dei primi seguaci di Gesù con quelle attribuite a Gesù stesso. La preghiera, le rivelazioni soprannaturali sono due forme culturali particolarmente interessanti per comprendere aspetti centrali della vita di Gesù.

Nel libro tu presenti ad un vasto pubblico alcuni dei risultati della ricerca storica su Gesù e le diverse interpretazioni che sono state date della sua vita e delle sue azioni - Gesù mistico, taumaturgo, mago, profeta, predicatore attento ai problemi sociali del suo tempo. Come si procede dal punto di vista storico alla ricostruzione della vita di Gesù? Quali sono le correnti storiografiche più accreditate? Esistono anche altrove conflitti tra la ricerca scientifica e le chiese? 
I tre gruppi scientifici - molto diversi fra loro - che hanno a mio avviso maggiormente contribuito al rinnovamento degli studi su Gesù e sul cristianesimo antico sono l'Association pour l'Etude de la Littérature Apocryphe Chrétienne (AELAC) europea, il Jesus Seminar statunitense, e le tendenze europee e statunitensi che rileggono le origini cristiane in modo socio-antropologico. Queste tre correnti hanno suscitato problemi, proposto nuove fonti e nuovi paradigmi interpretativi che hanno costretto moltissimi a rinnovare problematiche, metodi e soluzioni. Personalmente, pur avendo forti legami con l'AELAC, sono legato alla terza tendenza. Ne sono testimonianza una cinquantina di articolo scritti insieme all'antropologa Adriana Destro e libri come Antropologia delle origini cristiane (Laterza 1995) e Come nasce una religione (Laterza 2000). Tuttavia, io sono in genere metodologicamente e storiograficamente onnivoro. Non mi piacciono le scuole e i loro gerghi.

Queste nuove tendenze sono diffuse anche tra l'esegesi italiana, ma poco nella teologia cattolica. Del resto, nonostante l'amplissimo e libero dibattito che si svolge nella Society of Biblical Literature, anche nelle chiese statunitensi l'esegesi scientifica è poco recepita.

Contrariamente a quanto è stato affermato nelle polemiche recenti, il Gesù delle tue ricerche fa riemergere la complessa figura che si viene a cristallizzare nella memoria delle diverse comunità cristiane. Come avveniva la trasmissione della memoria di Gesù per i primi cristiani? Cosa leggevano e cosa conoscevano le prime comunità cristiane della vita, degli insegnamenti e delle azioni di Gesù?

La memoria di Gesù nelle prime comunità di discepoli di Gesù avveniva in modi fortemente differenziati. Agli inizi, la trasmissione dei fatti e delle parole di Gesù avveniva in ambienti ebraici i quali fornivano il contesto istituzionale religioso di base. Solo quando questo contesto ebraico in diverse zone e in epoche diverse cominciò ad allentarsi, divenne primaria l'esigenza di fondare soprattutto in Gesù le usanze religiose, liturgiche, la prassi morale e 
ideologica dei gruppi. È in questa fase che i vangeli adempiono ad una funzione importantissima. Nelle mie risposte ad Augias ho detto brevemente quello che avevo ipotizzato in un mio saggio e cioè che paradassalmente i vangeli - che ci permettono l'accesso privilegiato alla conoscenza di Gesù - ne costituiscono anche una prima forma di de-giudaizzazione e di cristianizzazione. Ma questa affermazione va intesa all'interno degli studi specializzati sui modi di trasmissione dei materiali relativi a Gesù e non come una svalutazione del significato e valore storico dei vangeli canonici. È comunque molto difficile stabilire con precisione cosa le singole comunità dei seguaci di Gesù leggessero, quali libri protocristiani usassero e quali testi ebraici. La trasmissione orale della parole e dei fatti di Gesù rimase viva almeno fino alla metà del II secolo con un valore anche più rilevante di quello che avevano opere scritte come i quattro vangeli divenuti poi canonici, i quali solo alla fine del II secolo cominciarono ad essere considerati più importanti degli altri.

Tu hai spesso sostenuto che il cristianesimo delle origini è un fenomeno culturale e religioso differenziato. Si parla spesso di diverse forme di cristianesimi. Come si è diffuso il cristianesimo nell'Impero romano? Qual è il rapporto tra un movimento religioso apparso nel mondo ebraico della Terra d'Israele e la sua diffusione negli ambienti urbani dell'Impero romano?

La tua domanda riguarda in fondo la grande questione della nascita del cristianesimo che comincia a formarsi solo dopo che le comunità dei discepoli di Gesù non fecero più parte della comunità dei Giudei. La mia affermazione che il cristianesimo come lo intendiamo noi potrebbe essere nato solo nella seconda metà del II secolo ha suscitato un enorme reazione da parte di Cantalamessa e di De Rosa, ma si tratta di un'affermazione storiografica ben articolata che è molto diffusa tra gli studiosi di oggi e non contiene nulla di contrario al cristianesimo. Nel 2004, un numero intero della rivista «Annali di Storia dell'Esegesi» dal titolo Come è nato il cristianesimo? è stato dedicatto a questo tema e studiosi di ogni parte di Europa prendono 
posizione a favore di una nascita tarda. Lo scandalo nasce solo da una insufficiente informazione del dibattito. In ogni caso, al centro del problema stanno tre fatti: Gesù era un ebreo perciò si pone neccssariamente la questione del quando il cristianesimo sia nato. Secondo: all'inizio esiste una molteplicità di versioni diverse della fede in Gesù e solo dopo la seconda metà del II secolo si afferma un cristianesimo normativo. Terzo: con il diffondersi nell'Impero romano ed in altre parti del mondo antico, le comunità di seguaci di Gesù non furono più composte da Ebrei e questo comportò una sostanziale modifica. L'interpretazione dell'incrociarsi di questi tre fattori costituisce un grande problema storiografico che è oggi molto dibattuto e lontano dal vedere un consenso nelle soluzioni.

\section{La "questione religiosa" diventerà, forse, una questione di primaria} importanza nei prossimi decenni. Non si tratterà solo del rapporto tra cristianesimi e società, ma anche di quello tra cristianesimi, società moderna e altre religioni. Come vedi questo rapporto anche alla luce delle vicende attuali sul tuo libro?

La questione religiosa è certo al centro del nostro futuro, anche se non dobbiamo mai dimenticare che spesso i fattori che la determinano non sono primariamente religiosi. Mi sono convinto da tempo che le religioni, dalla fine del Settecento in poi, si sono differenziate al proprio interno in diverse correnti che si diversificano per il modo con cui rispondono ad alcuni grandi problemi posti dalla cultura contemporanea i quali sono, a mio avviso: la presa di coscienza dei diritti naturali dei singoli, la scienza moderna e la sua spiegazione del mondo, lo studio storico delle religioni e i nuovi modi di vita sviluppati nelle grandi capitali culturali del mondo. Esiste un ventaglio di risposte religiose che va dall'accettazione di questi elementi culturali al loro radicale rifiuto attraverso tutta una serie di posizioni intermedie di compromesso. Lo studio storico di Gesù è uno dei frutti dello studio storico delle religioni e il dibattito pubblico sui fondamenti del cristianesimo rientra in quella caratteristica tipica delle società contemporanee fondate sui diritti naturali dei singoli, caratteristica che consiste nella cosiddetta "società 
civile", uno spazio neutro in cui tutti possono esprimere liberamente le proprie idee religiose, areligiose o irreligiose e non connotato, quindi, da una religione in particolare. L'università - fondata sul metodo scientifico - è il frutto tipico della società civile. La Chiesa cattolica ha impiegato circa settanta anni dal 1893 in poi (data della Enciclica di Leone XIII, Providentissimus Deus) per accettare lo studio storico della Bibbia, ma poi negli anni '80 e '90 del Novecento ha conosciuto il ritorno di una teologia non so quanto maggioritaria nel mondo cattolico - che nega o attenua molto l'interpretazione storica.

II fatto è che l'atteggiamento della teologia islamica è completamente ostile ad una interpretazione storica del Corano e della religione islamica nel suo nucleo profetico fondante. Di fronte all'irrigidimento fondamentalista di gran parte della teologia islamica le altre religioni stanno subendo un parallelo e contrapposto irrigidimento. II riconoscimento dello spazio neutro della società civile tende così a diminuire da parte delle grandi religioni mondiali che desidererebbero occupare un sempre maggior numero di spazi sociali. 